\title{
Smaller and larger deletions of the Williams Beuren syndrome region implicate genes involved in mild facial phenotype, epilepsy and autistic traits
}

\author{
Carmela Fusco $^{1,6}$, Lucia Micale ${ }^{1,6}$, Bartolomeo Augello ${ }^{1}$, Maria Teresa Pellico ${ }^{1}$, Deny Menghini ${ }^{2}$, \\ Paolo Alfieri ${ }^{2}$, Maria Cristina Digilio ${ }^{3}$, Barbara Mandriani ${ }^{1,4}$, Massimo Carella ${ }^{1}$, Orazio Palumbo ${ }^{1}$, \\ Stefano Vicari ${ }^{2}$ and Giuseppe Merla ${ }^{\star, 1,5}$
}

Williams Beuren syndrome (WBS) is a multisystemic disorder caused by a hemizygous deletion of $1.5 \mathrm{Mb}$ on chromosome 7q11.23 spanning 28 genes. A few patients with larger and smaller WBS deletion have been reported. They show clinical features that vary between isolated SVAS to the full spectrum of WBS phenotype, associated with epilepsy or autism spectrum behavior. Here we describe four patients with atypical WBS 7q11.23 deletions. Two carry $\sim 3.5 \mathrm{Mb}$ larger deletion towards the telomere that includes Huntingtin-interacting protein 1 (HIP1) and tyrosine 3-monooxygenase/tryptophan 5-monooxigenase activation protein gamma (YWHAG) genes. Other two carry a shorter deletion of $\sim 1.2 \mathrm{Mb}$ at centromeric side that excludes the distal WBS genes BAZ1B and FZD9. Along with previously reported cases, genotype-phenotype correlation in the patients described here further suggests that haploinsufficiency of HIP1 and YWHAG might cause the severe neurological and neuropsychological deficits including epilepsy and autistic traits, and that the preservation of $B A Z 1 B$ and $F Z D 9$ genes may be related to mild facial features and moderate neuropsychological deficits. This report highlights the importance to characterize additional patients with 7q11.23 atypical deletions comparing neuropsychological and clinical features between these individuals to shed light on the pathogenic role of genes within and flanking the WBS region.

European Journal of Human Genetics (2014) 22, 64-70; doi:10.1038/ejhg.2013.101; published online 12 June 2013

Keywords: Williams Beuren syndrome; 7q11.23; haploinsufficiency; qPCR

\section{INTRODUCTION}

The $1.5-1.8 \mathrm{Mb}$ hemizygous deletion of roughly 28 annotated genes at 7 q11.23 region causes Williams Beuren syndrome (WBS; OMIM number 194050). WBS patients display a characteristic pattern of symptoms including typical facial dysmorphism, supravalvular aortic stenosis (SVAS), weakness of connective tissue, short stature, mild-tomoderate intellectual disability (ID), and a characteristic cognitive profile that includes relative strengths in verbal short term memory and lexical comprehension, alongside severe weakness in visuospatial construction. Other clinical features include growth retardation, hyperacusis, premature ageing, hypercalcemia, glucose intolerance, renal anomalies, dental defects, gastrointestinal problems, urinary tract abnormalities, weakness in daily living skills, and motor

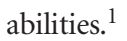

The dissection of the WBS phenotype relies mainly on evidences from functional studies of single genes, animal models, and analysis of WBS individuals with atypical deletions. These studies suggest correlations between haploinsufficiency of some WBS genes and WBS phenotypic features. For instance, reduced BAZ1B protein level has been linked to cardiac, craniofacial, and hypercalcemia defects; hemizygosity of LIMK1, CLIP2, GTF2IRD1, and GTF2I is associated to the specific WBS cognitive profile and craniofacial features. ${ }^{2,3}$

Here we describe four patients with atypical 7q11.23 deletions and complex WBS phenotypes. Two carry $\sim 3.5 \mathrm{Mb}$ larger deletion towards the telomere that includes Huntingtin-interacting protein 1 (HIP1) and tyrosine 3-monooxygenase/tryptophan 5-monooxigenase activation protein gamma (YWHAG). The other two carry shorter deletions of $\sim 1.2 \mathrm{Mb}$ at centromeric side that do not include the more distal WBS genes.

\section{SUBJECTS AND METHODS}

Subjects and clinical evaluation

Blood samples were obtained from proband and available parents after acquiring informed consent approved by the institutional review board. In WBS154 and WBS166 patients, the global cognitive evaluation was conducted by the Wechsler Intelligence Scale for Children Third Edition (WISC-III). ${ }^{4}$ As WBS160 had a severe ID and WBS179 was very young, both were not able to complete the WISC-III, and therefore their global cognitive abilities were assessed by using the Leiter International Performance Scale-Revised ${ }^{5}$ and the Griffiths Scales of Mental Development), ${ }^{6}$ respectively.

${ }^{1}$ Medical Genetics Unit, IRCCS Casa Sollievo Della Sofferenza Hospital, San Giovanni Rotondo, Italy; ${ }^{2}$ Child NeuroPsychiatry Unit, Neuroscience Department, IRCCS Children Hospital Bambino Gesù, Rome, Italy; ${ }^{3}$ Medical Genetics, IRCCS Children Hospital Bambino Gesù, Rome, Italy; ${ }^{4}$ PhD Program, Biomedical Sciences and Translational Medicine, University of Brescia, Brescia, Italy; ${ }^{5} \mathrm{PhD}$ Program, Reproduction and Development Sciences, University of Trieste, Trieste, Italy

${ }^{6}$ These authors are joint first authors.

*Correspondence: Dr G Merla, Medical Genetics Unit, IRCCS Casa Sollievo della Sofferenza, Poliambulatorio Giovanni Paolo II, San Giovanni Rotondo (FG) I-71013, Italy. Tel: +39 0882 416350, Fax: +39 0882 411616, E-mail: g.merla@operapadrepio.it

Received 12 December 2012; revised 6 March 2013; accepted 10 April 2013; published online 12 June 2013 
Complete evaluation concerning linguistic (production and comprehension), visuo-motor, praxic, coordination, adaptive abilities, and behavior aspects was reported for all the patients (Table 1).

\section{Multiple-ligation probe amplification and quantitative real-time PCR}

The multiple-ligation probe amplification (MLPA) analysis was performed with SALSA kit P029 (MRC Holland, http://www.mlpa.com), according to the manufacturer's instructions. Quantitative real-time PCR (qPCR) was carried out as described, ${ }^{3}$ with oligo pairs reported in Supplementary Table S2 and based on the UCSC GRCh37/hg19 assembly.

\section{CytoScan HD array and junctions fragment mapping}

The CytoScan HD assay was performed according to the manufacturer's protocol. Briefly, genomic DNA was digested, ligated to an adapter, and subjected to PCR amplification. After DNase I digestion, PCR products were labeled and hybridized. After that washing and staining were performed using the Fluidics Station 450 (Affymetrix, Santa Clara, CA, USA). The array was then scanned with the Scanner 3000 7G (Affymetrix), and both quality control step and copy number analysis were performed using the Chromosome Analysis Suite Software (Affymetrix): (i) the raw data file (CEL) was normalized using the default options; (ii) an unpaired analysis was performed using as baseline the 270 HapMap samples in order to obtain copy numbers value from CEL files while the amplified and/or deleted regions were detected using a standard Hidden Markov Model method. The Database of Genomic Variants (http:// www.projects.tcga.ca/variation/) was used to compare findings with previously reported studies. Coordinates of copy number variants, deletion breakpoints and oligo pairs used in qPCR are based on the UCSC GRCh37/hg19 assembly.

\section{RESULTS}

Neuropsychological and clinical features of WBS patients are summarized in Table 1 and Supplementary Table S1, respectively.

\section{Patient WBS160}

The proband, a 14.6-year-old female, is the only child of healthy nonconsanguineous parents. At birth, the mother was 32 years old, the father 34. The patient was born by vaginal delivery at term of an uneventful pregnancy. Birth weight was $2700 \mathrm{~g}$, length $48 \mathrm{~cm}$, and head circumference $34 \mathrm{~cm}$. Phenotypic examination showed truncal obesity, microcephaly, strabismus, characteristic WBS facial anomalies (for example, periorbital fullness, epicanthal folds, stellate iris, malar flattening, short nose with anteverted nares, long philtrum, large mouth with thick lips), small teeth with dental malocclusion, and scoliosis. Weight was $70 \mathrm{~kg}$ (90th centile), height $148 \mathrm{~cm}(<3 \mathrm{rd})$, and head circumference $50 \mathrm{~cm}(<3 \mathrm{rd})$. The voice was coarse. Feeding difficulties were referred during the first year of life. Hyperacusia was present. Blood calcium dosage was normal. Screening for celiac disease was negative. She was pharmacologically treated for tonicoclonic epilepsy from the age of 2 years with antiepileptics (actually with Lamotrigine). Cerebral and renal ultrasound examinations, cerebral MRI, 2-dimensional color-Doppler echocardiography were normal. Neuropsychological profile was characterized by a severe ID with diffuse neuropsychological deficits (Table 1 and Supplementary Table S1). Moreover, anxiety and obsessive behavior were documented by the psychopathological examination.

\section{Patient WBS179}

The proband, a 5.5-year-old female, is the second child of healthy non-consanguineous parents (Figure 1a). At birth, the mother was 33 years old, the father 34 . The patient was born by cesarean section at term of pregnancy. Ultrasound examinations after the 34th week showed intrauterine growth retardation. Birth weight was $2840 \mathrm{~g}$, length $47 \mathrm{~cm}$, and head circumference $33 \mathrm{~cm}$. Apgar scores were 6 and 8 at 1 and $5 \mathrm{~min}$, respectively. Congenital heart defect was diagnosed shortly after birth. Echocardiography showed valvular and

Table 1 Scores of WBS subjects on the neuropsychological testing

\begin{tabular}{|c|c|c|c|c|c|c|c|c|}
\hline & WBS154 & & WBS166 & & WBS 160 & & WBS179 & \\
\hline & CA: 15.0 y.m & & CA: 6.6 y.m & & CA: 14.6 y.m & & CA: 5.5 y.m & \\
\hline Total IQ or $D Q^{*}$ & $\begin{array}{l}\text { MA: } 6.6 \text { y.m } \\
43 \text { (WISC-III) }\end{array}$ & & $\begin{array}{l}\text { MA: } 3.0 \text { y.m } \\
46 \text { (WISC-III) }\end{array}$ & & $\begin{array}{l}\text { MA: } 3.0 \text { y.m } \\
36 \text { (LIPS-R) }\end{array}$ & & $\begin{array}{l}\text { MA: } 3.3 \text { y.m } \\
\text { *39 (GSMD) }\end{array}$ & \\
\hline Verbal IQ & 50 & & 56 & & & & & \\
\hline \multirow[t]{2}{*}{ Performance $I Q$} & 49 & & 48 & & & & & \\
\hline & $y \cdot m$ & Difference from $M A$ & $y . m$ & Difference from $M A$ & $y \cdot m$ & Difference from $M A$ & $y \cdot m$ & Difference from $M A$ \\
\hline Lexical production $^{a}$ & 10.0 (BNT) & +3.6 & 6.0 (BNT) & +3.0 & 3.6 (BNT) & +0.6 & $1.5(\mathrm{CDI})$ & -1.8 \\
\hline Lexical comprehensiona ${ }^{a}$ & 11.0 (PPVT) & +4.6 & 4.5 (PPVT) & +1.5 & 4.0 (PPVT) & +1 & $1.5(\mathrm{CDI})$ & -1.8 \\
\hline Sentence comprehension ${ }^{a}$ & NE & NE & $5.8(\mathrm{GCT})$ & +2.8 & $3.0(\mathrm{GCT})$ & 0 & $2.0(F L T)$ & -1.3 \\
\hline Nonverbal abilities ${ }^{a}$ & 5.6 (VMI) & -1.0 & $4.10(\mathrm{VMI})$ & +1.10 & 5.0 (PCET) & +2 & $2.0(\mathrm{VMC})$ & -1.3 \\
\hline \multicolumn{9}{|l|}{ Adaptive Abilities (VABS) } \\
\hline Communication ${ }^{a}$ & 13.2 & +7.2 & 6.6 & +3.6 & 3.11 & +0.11 & 2.2 & -1.1 \\
\hline Daily livinga & 3.1 & -3.5 & 8.3 & +5.3 & 2.11 & -0.1 & 2.8 & -0.5 \\
\hline Socialization ${ }^{a}$ & 7.10 & +1.4 & 8.0 & +5.0 & 3.2 & +0.2 & 2.2 & -2.2 \\
\hline Motor skillsa & NE & $\mathrm{NE}$ & 5.2 & +3.2 & 2.6 & -0.6 & 3.3 & 0 \\
\hline
\end{tabular}

Abbreviations: BNT, Boston Naming Test; CDI, MacArthur-Bates Communicative Development Inventory; DQ, developmental quotient; FLT, first language test; GCT, grammar comprehension test; GSMD, Griffiths Scales of Mental Development; IQ, intelligence quotient; LIPS-R, Leiter International Performance Scale-Revised; NE, not explored; PCET, Praxic and Coordination Evaluation Test; PPVT, Peabody Picture Vocabulary Test; VMI. Developmental Test of Visuo-Motor Integration; WISC-III, Wechsler Intelligence Scale for Children Third Edition; VABS, Vineland Adaptive Behavior Scales; VMC, the Visual Manual Coordination subtest of the Griffiths Scales of Mental Development.

Deviation from mental age (MA) is expressed in year and month (y.m).

${ }^{a}$ All scores are age-corrected scaled scores and expressed in equivalent age. 
a
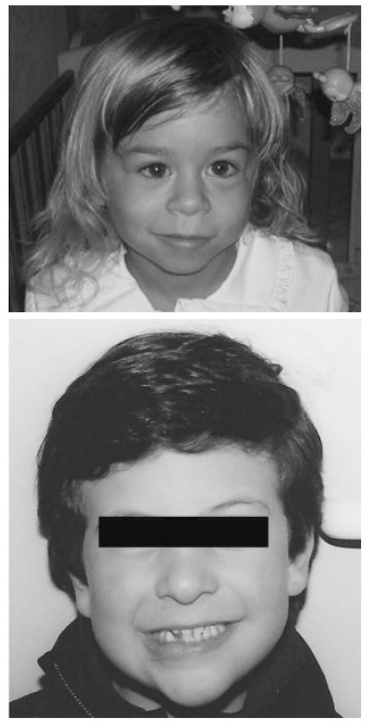
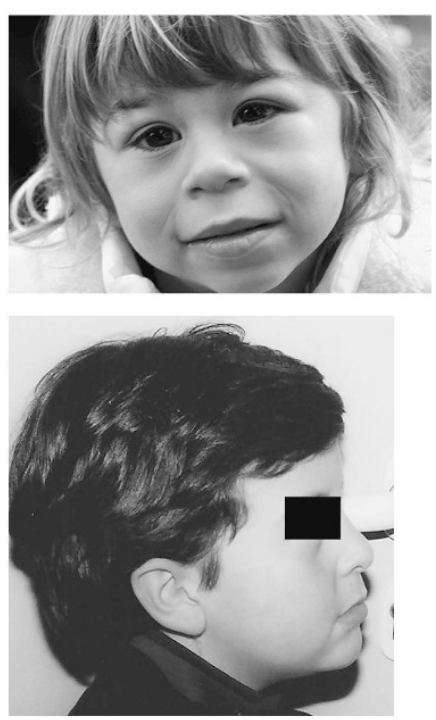

b

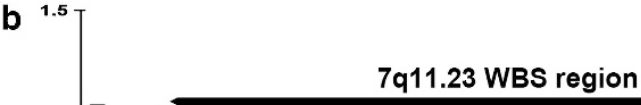

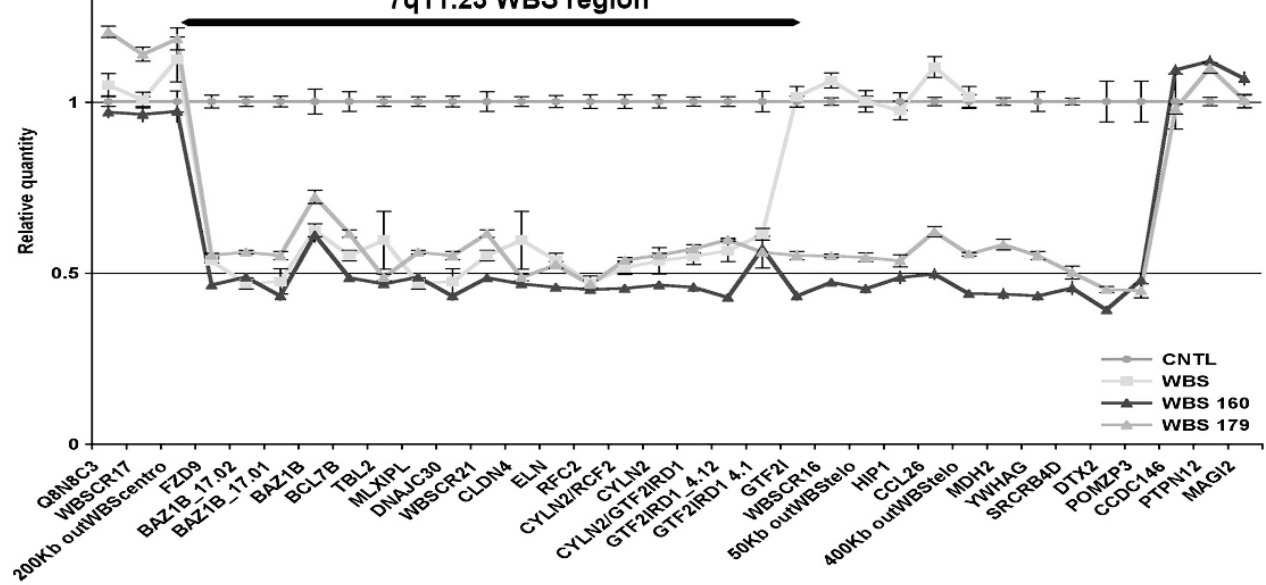

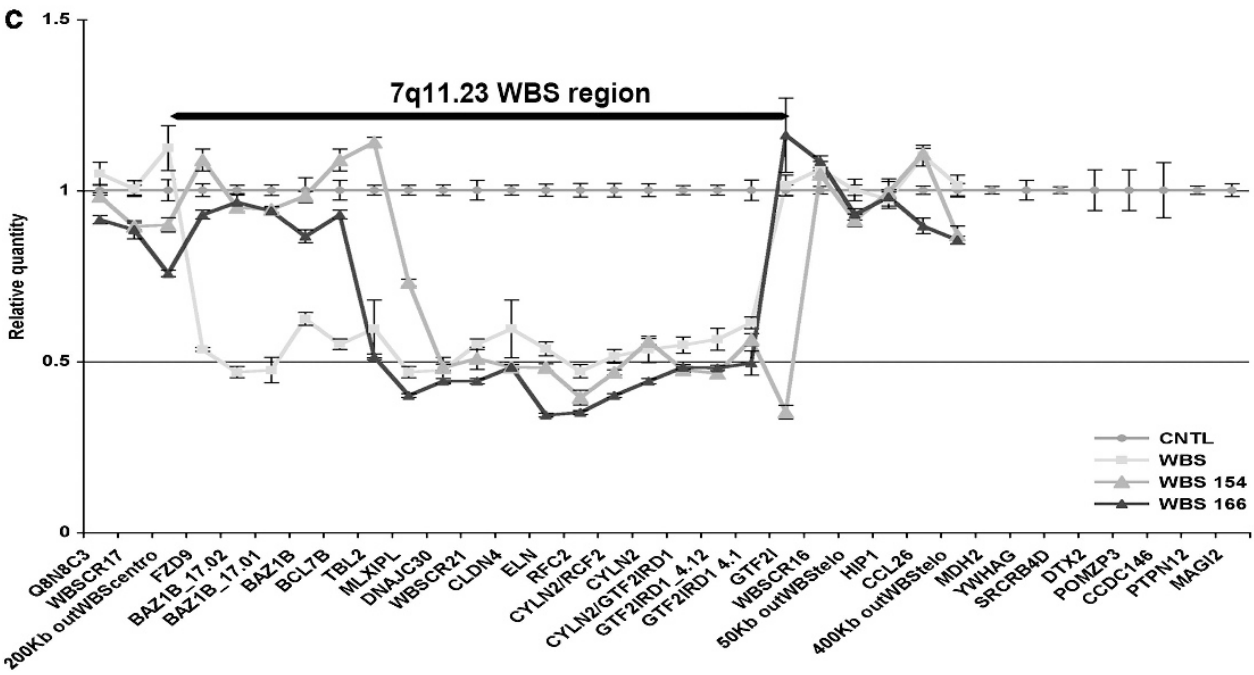

Figure 1 Facial and molecular features of representative WBS patients. (a) Representative images of facial characteristics of WBS patient carrying larger (WBS 179, top) and smaller deletion (WBS 166, bottom). (b, c) Mapping WBS atypical deletion by qPCR. Relative DNA quantity was quantified for the four probands. (b) shows patients with largest deletion (WBS 179 and WBS 160) and (c) those with smallest ones (WBS 154 and WBS166) as well as an unaffected control (CNTL) and a WBS patient carrying the classical deletion (WBS). 
supravalvular pulmonary stenosis with mild peripheral pulmonary stenosis. Feeding difficulties were referred during the first 2 years of life. Cerebral and renal ultrasound examinations were normal. Bilateral convergent strabismus was operated at 4 years of age. Developmental milestones were retarded. The baby was able to walk alone at 27 months; first words appeared at 42 months. Phenotypic examination showed microcephaly, typical facial anomalies of WBS (periorbital fullness, epicanthal folds, stellate iris, flat nasal bridge, anteverted nares, large mouth with thick lips), small teeth, and umbilical hernia. Weight was $13500 \mathrm{~kg}$ ( $<3$ rd centile), height $101 \mathrm{~cm}$ (3rd-10th centile), and head circumference $47.5 \mathrm{~cm}$ ( $<3 \mathrm{rd}$ centile). Echocardiography at 5 years of age showed mild suprapulmonary stenosis. Blood calcium dosage was normal. Neuropsychological profile was characterized by a severe ID with diffuse deficits compared with her mental age. Behavior was characterized by aggressivity, acts of self-injury, social communication problems, stereotypes, hyperactivity/impulsivity, and temper tantrums. On child behavior checklist, ${ }^{7}$ this patient obtained clinical results on syndromic scale 'withdrawn' and 'pervasive developmental disorder'. Critical scores for the pervasive developmental disorder derived also from the Social Communication Questionnaire (total score $>15)^{8}$ and for an attention deficit and hyperactivity disorder from the Conners Scale (T score 90). ${ }^{9}$

\section{Patient WBS154}

The proband, a 15-year-old female, is the first child of healthy nonconsanguineous parents. Family history was unremarkable. At birth, the mother was 32 years old, the father 35 . The baby was born by cesarean section at the 42th week of an uneventful gestation. Birth weight was $3460 \mathrm{~g}$, length $50.5 \mathrm{~cm}$, and head circumference $34.4 \mathrm{~cm}$. Apgar scores were 8 and 9 at 1 and $5 \mathrm{~min}$, respectively. Developmental milestones were retarded. Two-dimensional echocardiography and renal ultrasound examination were normal. Phenotypic examination at 15 years showed mild facial features of WBS, including mild malar hypoplasia and thick lips. Epicanthal folds, flat nasal bridge, stellate iris, anteverted nares, large mouth, and small teeth were not present in this patient. Weight was $52 \mathrm{~kg}$ (25th-50th centile), height $160 \mathrm{~cm}$ (25th-50thcentile), and head circumference $54 \mathrm{~cm}$ (25th-50th centile). Strabismus was treated with eye occlusion and lens. Kyphosis and subluxation of both rotulae were noted at 13 years of age. Screening for celiac disease was negative. Abdominal ultrasound examination and echo-Doppler ultrasound of renal vessels were all normal. Neuropsychological profile was characterized by a moderate ID with neuropsychological functioning characterized by linguistic abilities and some features of adaptive abilities (that is, communication and socialization) more proficient than those predictable on the basis of her mental age (Table 1). Moreover, anxiety and attention deficit hyperactivity disorder were also found.

\section{Patient WBS166}

The proband, a 6.6-year-old male, is the third child of healthy nonconsanguineous parents (Figure 1a). An older brother was deceased after cardiac surgery for transposition of the great arteries with ventricular septal defect. At birth, the mother was 29 years old, the father 31. The proband was born by cesarean section at term of an uneventful pregnancy. Birth weight was $3200 \mathrm{~g}$, length $50.5 \mathrm{~cm}$, and head circumference $35 \mathrm{~cm}$. Apgar scores were 8 and 9 at 1 and $5 \mathrm{~min}$, respectively. Congenital heart defect was diagnosed in the first days of life. Echocardiography showed SVAS, which was operated at 12 months of age, and mild peripheral pulmonary stenosis. At 3 years of age, hypertension needing pharmacological treatment was diagnosed. Developmental milestones were retarded. At 6 years, weight was $28 \mathrm{~kg}$ (90th centile), height $126 \mathrm{~cm}$ (90th-97th centile), and head circumference $49.8 \mathrm{~cm}$ (10th centile). Phenotypic examination showed mild atypical features of WBS, including flat face, narrow palpebral fissures, malar hypoplasia, thick everted lower lip, low-set ears, and narrow shoulders. Neuropsychological profile was characterized by a moderate ID (Supplementary Table S1) with a proficient neuropsychological profile especially when compared with his mental age (Table 1). Specifically, scores obtained in verbal, nonverbal, and adaptive tasks were above his mental age level. Finally, no psychopathological disorder was observed.

\section{Deletion mapping}

FISH and MLPA analysis confirmed WBS clinical diagnosis by detecting a de novo heterozygous deletion at chromosome 7q11.23 for all four patients (data not shown and Supplementary Figure S1). Notably, MLPA results were indicative of smaller atypical deletions in WBS154 and WBS166 samples. aCGH and qPCR were carried out to precisely map the extents of the deletion (Figure 1 and Supplementary Table S3) and to exclude the contribution of other copy number variants (CNVs) to the observed phenotypes. No additional pathological CNVs were identified in all four patients, suggesting that the observed complex phenotypes are consequences of the 7q11.23 deletion. The deletion of WBS160 and WBS179 patients showed a similar extension. Both are heterozygous for the common WBS deletion, encompassing the proximal $1.43 \mathrm{Mb}$ of the Williams Beuren Syndrome Chromosomal Region (WBSCR) but extending to $\sim 2 \mathrm{Mb}$ beyond it, with CCDC146 as the first preserved two copies-gene located distally to WBSCR deletion (Figure $1 \mathrm{~b}$ and Supplementary Table S3). RepeatMasker analysis of the regions flanking each deletion breakpoint showed that the sequence immediately upstream and downstream the WBS160 deleted region fell within a simple tandem repeat $5^{\prime}$-(CTTTAAGAAAAGAAACG)- $3^{\prime}$ mapping inside the STAG3L3 gene and within the long interspersed nuclear element (LINE) and short interspersed elements (SINE) repeats, respectively. Similarly analysis for the WBS179 revealed an enrichment of LINE and SINE repeats at the distal breakpoint junction and the presence of a L1MC3 element (LINE L1 family element) at the proximal breakpoint.

The minimum deletion for WBS154 was $1.04 \mathrm{Mb}$ in size (chr7: $73092574-74141493$ ) spanning the whole WBSCR distal to ELN, including GTF2I, and preserving the TBL2 gene at the proximal end of the deletion. At the sequence level, we found that the breakpoints of the region implicated in the WBS154 rearrangements were located in SINE dense regions.

WBS166 carried a 1.03-Mb deletion (chr 7:72 974959-74005314) with an intact $B C L 7 B$ gene at the centromeric end of WBSCR and preserving GTF2I at the telomeric end (Figure 1c and Supplementary Table S3). Analysis of the sequence flanking the distal breakpoint revealed an enrichment of SINE elements within GTF2IRD1 gene, whereas the proximal breakpoint was found close to Alu elements.

\section{DISCUSSION}

WBS patients with uncommon deletion size at $7 \mathrm{q} 11.23$ are of particular interest for WBS genotype-phenotype correlation (Figure 2). In particular, larger WBS deletions extending distally show a more severe developmental delay (DD) or ID phenotype with serious impairments in cognitive function sometimes accompanied by infantile spasms and epilepsy, infrequently diagnosed in WBS patients with common deletion. ${ }^{10}$ In this study, we identified two WBS patients with larger deletions towards the telomere. Both patients 


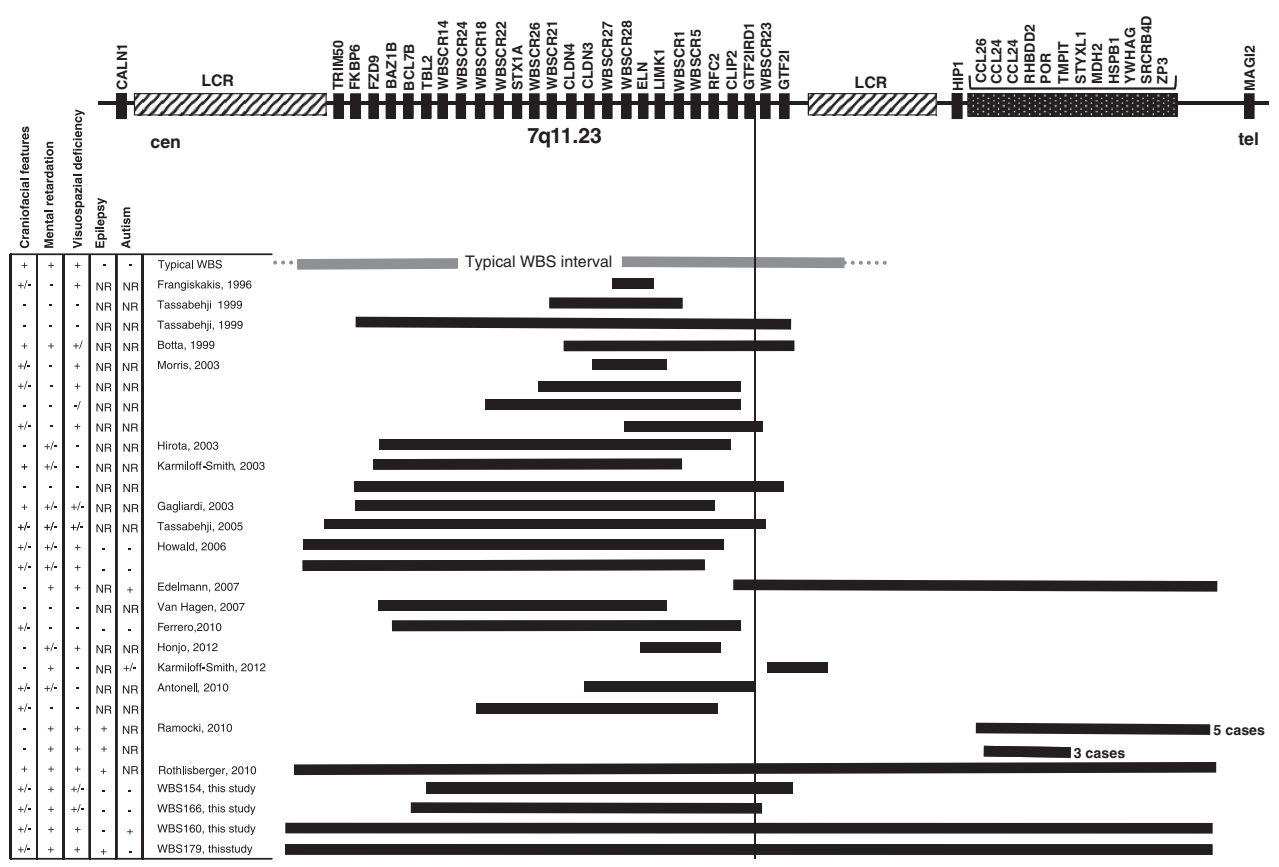

Figure 2 Typical and atypical 7q11.23 deletions in WBS patients along with main clinical information. A schematic representation of the genes mapping at 7q11.23 critical region is depicted on the top of the figure. Classical and atypical WBS intervals are depicted as gray and black solid lines, respectively. Main clinical features are described in the box. NR, not reported.

presented severe DD, ID, and neuropsychological deficits. WBS179 showed recurrent self-injurious acts, impulsivity, hyperactivity, aggression, and autistic traits, as reported by SCQ test, while WBS160 was pharmacologically treated for tonico-clonic epilepsy. Among the genes deleted distally to the WBS common deletion interval, HIP1 and YWHAG have been proposed as the most compelling candidate genes for susceptibility to autistic traits, epilepsy, and ID. ${ }^{10}$ In a loss-of-function model, mice with targeted mutation in the Hip1 gene developed a neurological phenotype, characterized by failure to thrive, tremor, gait ataxia, and epilepsy. ${ }^{11}$ Moreover, Hipl is crucial for $\alpha$-amino-3-hydroxy-5-methyl-4isoxazolepropionic acid (AMPA) and $N$-methyl-D-aspartate (NMDA) receptor trafficking in primary hippocampal neurons whose defects are associated with major progressive neurological deficits. ${ }^{12}$ Thus, the haploinsufficiency of HIP1 is sufficient to alter neuronal homeostasis predisposing the human brain to epilepsy through a mechanism involving abnormal AMPA and NMDA iGluR trafficking.

YHWAG is a member of a highly conserved family of phosphopeptide-binding proteins involved in signal transduction, protein localization, cell-cycle checkpoint control, and apoptosis. ${ }^{13}$ The loss of function of zebrafish ywhagl leads to brain DD and heart tube enlargement, indicating that YWHAG may have a role in both brain and heart development. Moreover, based on ywhag1 knockdown phenotype and expression pattern, the hemizigosity of YWHAG has been proposed to contribute to epilepsy and cardiomegaly. ${ }^{14}$

In a recent study, Ramocki et al ${ }^{10}$ reported individuals with heterozygous microdeletions distally adjacent to the WBS region inclusive of $H I P 1$, but not $Y W H A G$ in patients with variable expression and/or incomplete penetrance of epilepsy, learning difficulties, intellectual disabilities, and/or neurobehavioral abnormalities, suggesting that deletion of HIP1 is sufficient to cause neurological disease. Together with these collective findings, our results led to the postulation that HIP1 and YWHAG genes might contribute to the WBS neurological dysfunction when deleted along with other WBS genes.

The comparison of the phenotype of both patients with smaller WBS deletion with that of classical WBS patients revealed some interesting clues. Both patients have a mild facial gestalt of WBS; they do not present stellate iris, short upturned nose, wide mouth, dental abnormalities, and the malocclusion commonly observed in WBS patients. Notably, three other fairly common WBS features as microcephaly, full lips, and long philtrum are not present in WBS154, suggesting that the genes proximal to $T B L 2$, including TRIM50, FKBP6, FDZ9, and BAZ1B, might be involved for the onset of such phenotypes. The cognitive profile investigation documented a moderate ID. Moreover, WBS154 and WBS166 resulted particularly proficient in language and communication skills and not only in lexical comprehension (Table 1). This neuropsychological profile with relatively intact language and, concerning adaptive behavior, strength in communication is in line with that observed in typically deleted WBS individuals. ${ }^{15-17}$

Careful analysis of facial features of patients with atypical deletion and varying degrees of craniofacial abnormalities and animal model studies suggest that genes at both proximal and distal ends of the deletion are involved in WBS craniofacial features. ${ }^{3,18-21}$ In particular, two genes have been postulated to be mainly involved in the craniofacial features of WBS, GTF2IRD1, and BAZ1B.

GTF2IRD1 was proposed as a key gene in facial morphogenesis based on the finding of reported craniofacial defects reminiscent of the WBS in a mouse lines homozygous for a chromosomal translocation disrupting the Gtf2ird1 gene. ${ }^{22}$ In addition, GTF2IRD1 regulates the goosecoid gene involved in craniofacial morphogenesis ${ }^{23}$ as well, as mice haploinsuficient for a mouse model carrying a deletion between Clip2 and Gtf2ird1 show hypoplasia of the mandible and other craniofacial defects resembling the defects and dental problems of WBS individuals. ${ }^{24}$ However, another targeted knockout of Gtf2ird1 failed to find craniofacial dysmorphology, ${ }^{25}$ suggesting 
that this phenotype may be influenced by different genetic background of diverse strains.

$B A Z 1 B$ was implicated in craniofacial development through a mouse model generated by random chemical mutagenesis resulting in the heterozygous L733R change in a highly conserved amino acid. Although this mouse line is not a true knockout, and thus we cannot know a priori whether it recapitulates the assumed Bazlb haploinsufficieny seen in WBS cell lines, this substitution resulted in reduced protein level compared with its wild-type countepart. ${ }^{26}$ Notably, mice homozygous for this mutation died within the first week of life, similar to the Bazlb knock-out mice described inYoshimura et al. ${ }^{27}$ Bazlb heterozygote mice present slightly narrower and shorter craniums compared with wild-type mice as well as reduced size of the posterior region of the lower jaw, indicating a role for $B a z 1 b$ in the craniofacial development. Moreover, the reduction of the Bazlb protein level produces some craniofacial features similar to those shown by typical WBS patients, such as a small upturned nose with flat nasal bridge, malocclusion, bitemporal narrowing, and prominent forehead. ${ }^{26}$ Notably, WBS154 and WBS166, both with two copies of $B A Z 1 B$, do not present any of the above features, suggesting that $B A Z 1 B$ and genes mapping at the proximal end of the WBS deletion are tantalizing candidates for WBS facies.

Insights about WBS craniofacial features come from full-deletion mice model. Although the most remarkable abnormalities in craniofacial development are observed for mice lacking the entire WBS syntenic region, ${ }^{28}$ mice lacking the human proximal region spanning Trim50:Limk1 (that includes Bazlb) showed only very mild facial defects. On the contrary, no differences in cranial morphology were reported in mice lacking the human distal region (Limk1:Gtf2i), in contrast to reported cases of individuals with atypical deletions that postulate a role for the most WBSCR distal genes as involved in the craniofacial phenotype. .3,22,29 $^{2}$ Overall, these results highlight the complexity of craniofacial development and WBS.

FZD9 gene is selectively expressed in the hippocampus throughout life with a strong expression in the neuroepithelium. Fzd9 null and heterozygous mice have increased apoptotic cell death and increased precursor proliferation during hippocampus development. Moreover, Fzd9 null mutants exhibit defects in learning and memory, reflecting hippocampus functional deficits. ${ }^{30}$ Overall, these evidences point out that Fzd9 has an important role in hippocampus development, and therefore, Fzd9 may be an intriguing candidate for the neurodevelopmental and behavioral phenotype of WBS individuals. Overall, these observations suggest that $B A Z 1 b$ and FZD9 remain two good candidate genes for the facial features and neurodevelopmental phenotype of WBS patients, respectively.

At the sequence level, we found that the sequences surrounding the deleted regions in all cases analyzed container repetitive elements, reported to give rise to genomic deletions by promoting recombinational instability. Although the vast majority of WBS deletions result by nonallelic homologous recombination between large regions of very high identity, the nonrecurrent deletions are likely random events that could be facilitated by other repetitive elements flanking the deleted intervals. However, the location of the breakpoints in nonhomologous or with low similarity sequences suggests that the most likely mechanism leading to this atypical rearrangements may be nonhomologous end joining or other processes that do not required high homology at the breakpoint, and support the proposal that other mechanisms predisposes to genomic instability of the WBS region ${ }^{31}$

This study underscores the importance to map precisely the WBS deletion boundaries to revaluate the significance of the WBS genes hemizygosity in the pathogenesis of epilepsy, facial features, and neurological phenotypes. ${ }^{32-40}$

\section{CONFLICT OF INTEREST}

All patients and/or their parents gave written consent for the study, for genetic testing some gave consent for publication of photos. Ethics Committee approval was not required as this was not a specific study but a report of clinical investigation undertaken for four WBS patients, as part of their standard clinical care. The founders had no role in study design, data collection and analysis, decision to publish, or preparation of the manuscript.

\section{ACKNOWLEDGEMENTS}

We thank all the families that agreed to participate and get possible this study. This work was in part supported by grants from the Italian Ministry of Health (Ricerca Corrente 2008-10) and the Jerome Lejeune Foundation to GM. The GDB bank, member of the Telethon Network of Genetic Biobanks (Project No. GTB12001), funded by Telethon Italy, provided us with some WBS specimens.

\section{AUTHOR CONTRIBUTIONS}

CF, LM, and GM designed the study and conceived the experiments; CF, LM, MTP, B.A., and B.M. carried out the experiments and analyzed the data. M.C.D., P.A., D.M., M.C. and O.P. performed and interpreted aCGH, and S.V. recorded clinical phenotype and performed neuropsycological and behavioral tasks. C.F., L.M, and G.M. wrote the manuscript with the support of all other authors. All authors discussed the results and commented on the manuscript.

1 Merla G, Brunetti-Pierri N, Micale L, Fusco C: Copy number variants at WilliamsBeuren syndrome 7q11.23 region. Hum Genet 2010; 128: 3-26.

2 Antonell A, Del Campo M, Magano LF et al: Partial 7q11.23 deletions further implicate GTF2I and GTF2IRD1 as the main genes responsible for the WilliamsBeuren syndrome neurocognitive profile. J Med Genet 2010; 47: 312-320.

3 Ferrero GB, Howald C, Micale L et al: An atypical 7q11.23 deletion in a normal $1 Q$ Williams-Beuren syndrome patient. Eur J Hum Genet 2010; 18: 33-38.

4 Wechsler D: Manual for the Wechsler intelligence scale for Children. New York: Psychological Corporation, 1991

5 Roid G, Miller L: Leiter International Performance Scale-Revised: Examiner's Manual. Wood Dale, IL: Stoelting Co, 1997.

6 Griffiths R: Griffiths Mental Development Scales, 1996.

7 Achenbach TM: A RL: Manual for the ASEBA Preschool Forms \& Profiles, 2000.

8 Rutter M, Bailey A: C. L: Social Communication Questionnaire. Los Angeles, CA: Western Psychological Services, 2003.

9 Conners C: Conners' rating scales-revised. New York and Toronto: Multi-Health Systems, Inc., 2006

10 Ramocki MB, Bartnik M, Szafranski $P$ et al: Recurrent distal 7q11.23 deletion including HIP1 and YWHAG identified in patients with intellectual disabilities, epilepsy, and neurobehavioral problems. Am J Hum Genet 2010; 87: 857-865.

11 Metzler M, Gan L, Wong TP et al: NMDA receptor function and NMDA receptordependent phosphorylation of huntingtin is altered by the endocytic protein HIP1 J Neurosci 2007; 27: 2298-2308.

12 Meyer-Lindenberg A, Mervis CB, Sarpal D et al: Functional, structural, and metabolic abnormalities of the hippocampal formation in Williams syndrome. J Clin Invest 2005; 115: 1888-1895.

13 Gardino AK, Yaffe MB: 14-3-3 proteins as signaling integration points for cell cycle control and apoptosis. Semin Cell Dev Biol 2011; 22: 688-695.

14 Komoike $\mathrm{Y}$, Fujii K, Nishimura A et al: Zebrafish gene knockdowns imply roles for human YWHAG in infantile spasms and cardiomegaly. Genesis 2010; 48: 233-243.

15 Vicari S, Bates E, Caselli MC et al: Neuropsychological profile of Italians with Williams syndrome: an example of a dissociation between language and cognition? J Int Neuropsychol Soc 2004; 10: 862-876.

16 Mervis CB, Klein-Tasman BP: Williams syndrome: cognition, personality, and adaptive behavior. Ment Retard Dev Disabil Res Rev 2000; 6: 148-158.

17 Martens MA, Wilson SJ, Reutens DC: Research Review: Williams syndrome: a critical review of the cognitive, behavioral, and neuroanatomical phenotype. J Child Psychol Psychiatry 2008; 49: 576-608.

18 Botta A, Novelli G, Mari A et al: Detection of an atypical 7q11.23 deletion in Williams syndrome patients which does not include the STX1A and FZD3 genes. J Med Genet 1999; 36: 478-480.

19 Edelmann L, Prosnitz A, Pardo S et al: An atypical deletion of the Williams-Beuren syndrome interval implicates genes associated with defective visuospatial processing and autism. J Med Genet 2007; 44: 136-143. 
20 Hirota $\mathrm{H}$, Matsuoka R, Chen XN et al: Williams syndrome deficits in visual spatial processing linked to GTF2IRD1 and GTF2I on chromosome 7q11.23. Genet Med 2003; 5: 311-321.

21 Howald C, Merla G, Digilio MC et al: Two high throughput technologies to detect segmental aneuploidies identify new Williams-Beuren syndrome patients with atypical deletions. J Med Genet 2006; 43: 266-273.

22 Tassabehji M, Hammond P, Karmiloff-Smith A et al: GTF2IRD1 in craniofacial development of humans and mice. Science 2005; 310: 1184-1187.

23 Thompson PD, Webb M, Beckett W et al: GTF2IRD1 regulates transcription by binding an evolutionarily conserved DNA motif 'GUCE'. FEBS Lett 2007; 581: 1233-1242.

24 Enkhmandakh B, Makeyev AV, Erdenechimeg L et al: Essential functions of the Williams-Beuren syndrome-associated TFII-I genes in embryonic development. Proc Natl Acad Sci USA 2009; 106: 181-186.

25 Palmer SJ, Tay ES, Santucci N et al: Expression of Gtf2ird1, the Williams syndromeassociated gene, during mouse development. Gene Expr Patterns 2007; 7: 396-404.

26 Ashe A, Morgan DK, Whitelaw NC et al: A genome-wide screen for modifiers of transgene variegation identifies genes with critical roles in development. Genome Biol 2008; 9: R182.

27 Yoshimura K, Kitagawa H, Fujiki R et al: Distinct function of 2 chromatin remodeling complexes that share a common subunit, Williams syndrome transcription factor (WSTF). Proc Natl Acad Sci USA 2009; 106: 9280-9285.

$28 \mathrm{Li} \mathrm{HH}$, Roy M, Kuscuoglu U et al: Induced chromosome deletions cause hypersociability and other features of Williams-Beuren syndrome in mice. EMBO Mol Med 2009 1: 50-65.

29 Morris CA: Genotype-phenotype correlations in Williams-Beuren syndrome. Baltimore, MD: John Hopkins University Press, 2006

30 Zhao C, Pleasure SJ: Frizzled9 protein is regionally expressed in the developing medial cortical wall and the cells derived from this region. Brain Res Dev Brain Res 2005; 157: 93-97.
31 Lee JA, Carvalho CM, Lupski JR, DNA A: replication mechanism for generating nonrecurrent rearrangements associated with genomic disorders. Cell 2007; 131 1235-1247.

32 Frangiskakis JM, Ewart AK, Morris CA et al: LIM-kinase 1 hemizygosity implicated in impaired visuospatial constructive cognition. Cell 1996; 86: 59-69.

33 Gagliardi C, Bonaglia MC, Selicorni A, Borgatti R, Giorda R: Unusual cognitive and behavioural profile in a Williams syndrome patient with atypical 7q11.23 deletion. J Med Genet 2003; 40: 526-530.

34 Honjo RS, Dutra RL, Nunes MM et al: Atypical deletion in Williams-Beuren Syndrome critical region detected by MLPA in a patient with supravalvular aortic stenosis and learning difficulty. J Genet Genomics 2012; 39: 571-574.

35 Karmiloff-Smith A, Broadbent H, Farran EK et al: Social cognition in williams syndrome: genotype/phenotype insights from partial deletion patients. Front Psychol 2012; 3: 168 .

36 Karmiloff-Smith A, Grant J, Ewing S et al: Using case study comparisons to explore genotype-phenotype correlations in Williams-Beuren syndrome. J Med Genet 2003; 40: 136-140.

37 Morris CA, Mervis CB, Hobart HH et al: GTF2I hemizygosity implicated in mental retardation in Williams syndrome: genotype-phenotype analysis of five families with deletions in the Williams syndrome region. Am J Med Genet A 2003; 123: 45-59.

38 Rothlisberger B, Hoigne I, Huber AR, Brunschwiler W, Capone Mori A: Deletion of 7q11.21-q11.23 and infantile spasms without deletion of MAGI2. Am J Med Genet A 2010; 152A: 434-437.

39 Tassabehji M, Metcalfe K, Karmiloff-Smith A et al: Williams syndrome: use of chromosomal microdeletions as a tool to dissect cognitive and physical phenotypes. Am J Hum Genet 1999; 64: 118-125.

40 van Hagen JM, van der Geest JN, van der Giessen RS et al: Contribution of CYLN2 and GTF2IRD1 to neurological and cognitive symptoms in Williams Syndrome. Neurobiol Dis 2007; 26: 112-124.

Supplementary Information accompanies this paper on European Journal of Human Genetics website (http://www.nature.com/ejhg) 